DOI: 10.20472/BM.2019.7.1.001

\title{
GREEN CONCEPT EVALUATION THROUGH FUZZY AHP-PROMETHEE II
}

\section{ZEKI AYAĞ}

\begin{abstract}
:
The demand for green products have dramatically increased because the importance and public awareness of the preservation of natural environment was taken into consideration much more last two decades. As a result of this, especially manufacturing companies have been forced to design more green products, resulting in a problem of how they incorporate environmental issues into their design and evaluate concept options. The need for the practical decision making tools to address this problem is rapidly evolving due to the fact that the problem turns into a multiple-criteria decision making (MCDM) problem in the presence of a set of green concept alternatives and criteria. Therefore; in this paper, the four popular MCDM methods in fuzzy environment are utilized to reflect the vagueness and uncertainty on the judgments of DMs, because the crisp pairwise comparison in these conventional MCDM methods seems to be insufficient and imprecise to capture the right judgments of DMs. Of these methods; as Fuzzy AHP is used to calculate criteria weights, the other method; Fuzzy PROMETHEE II is used to rank alternatives. Furthermore, the incorporation of fuzzy set theory into these methods is discussed on a real-life case study.
\end{abstract}

\section{Keywords:}

New product development, green concept selection, multiple-criteria decision making, fuzzy logic, AHP, PROMETHEE II.

JEL Classification: C44, C00, D81

\section{Authors:}

ZEKI AYAĞ, Kadir Has University, Turkey, Email: zekia@khas.edu.tr

\section{Citation:}

ZEKI AYAĞ (2019). Green Concept Evaluation through Fuzzy AHP-PROMETHEE II. International Journal of Business and Management, Vol. VII(1), pp. 1-10., 10.20472/BM.2019.7.1.001 


\section{Introduction}

Designing green products as the result of raising demand from public awareness of the preservation of natural environment have become a critical concern for companies, incorporating environmental issues in their product design according to meeting recent green guidelines. For companies to follow these guidelines in their new product development environment has to be carried out with special procedures. A NPD process is the sequence of steps or activities which an enterprise employs to conceive, design and commercialize a product (Ulrich and Eppinger, 2000). This process has the following activities with environmental issues from raw materials, production, transportation and distribution to re-use, remanufacturing, recycling to final disposal (Zhang et. al., 1997); (a) identifying customer needs, (b) establishing target specifications, (b) concept generation, (d) concept selection, (e) concept testing, (f) setting final specifications, (g) project planning, (h) economic analysis, (i) benchmarking of competitive products, (j) modeling and (k) prototyping.

Among these activities; the concept selection is a process of evaluating a set of concept alternatives in terms of the criteria (i.e. quality level and unit cost) to find out the best option (Ayag, 2005a; 2005b). It is also critical because the selected concept plays important role at the phase of generating a set of the design alternatives. On the other hand, it is pointed out in literature that around $70 \%$ of the unit cost of a product is committed at this phase (Duffy et al., 1993). After this, the development process will lead to a more detailed solution. Therefore, the concept selection is shortly defined to evaluate a set of design alternatives in a new product environment, and also a critical element to improve design productivity. In addition, during the development process, a company's product engineers (or designers) have to consider an increased number of design options to meet the needs of customers. The activity of judging and selecting from a set of competing design options is referred to as evaluation. As the number of design options to evaluate increases and the time available decreases, it is clear that designers or product engineers needs more help evaluate the possible concept alternatives and determine the most satisfying one. So, the evaluation process can be defined as a multiple-criteria decision making (MCDM) problem due to the fact that there are a set of alternatives which should be evaluated in terms of evaluation criteria, and a decision-maker(s) (DMs) will need at least one of MCDM methods in current literature. Therefore; in this paper, the two popular MCDM methods are chosen for the evaluation design alternatives, such as Analytic Hierarchy Process (AHP) invented by Thomas L. Saaty (Saaty, 1981) and PROMETHEE II (Preference Ranking Organization Method for Enrichment Evaluations) by Jean-Pierre Brans (Brans et al., 1986).

On the other hand, these conventional MCDM methods use a crips scale to reach the best satisfying alternative. As result of this, some shortcomings are observed as follows: it causes unbalanced scale of the judgments of a DM, does not model the uncertainty by mapping of DM's judgment to a number, the subjective judgment of a DM has great influence on the ranking. Due to the vagueness and uncertainty on the judgments of a DM, the crisp comparison in these conventional methods seems to be insufficient and imprecise to capture the right judgments of DMs. That's why that, in this study, fuzzy logic is utilized to make up for this deficiency in the conventional methods.

Shortly, the objective of this paper is to propose a fuzzy AHP (F-AHP) based approach to green concept evaluation problem through Fuzzy PROMETHEE II (F-PROMETHEE II) methods. Of these methods; as F-AHP is used to calculate criteria weights, the other; F- PROMETHEE II are used to rank alternatives. The integration of fuzzy set theory into the two methods is discussed on a real-life case study.

\section{Proposed approach}

Designing a green product or components in a new product development (NPD) environment is a comprehensive process because the process is progressively detailed through a series of phases. At the end of each phase called generally "the gate"; a design review is held to approve the design and release it to the next level. In this paper, as one of the critical phases of the NPD process, the phase of concept selection is taken into consideration to evaluate green concept alternatives in order to find out the most appropriate green concept for further development activities. On the other hand, the selecting process for the best concept becomes so vital and complicated for companies because, as the development progresses on a selected concept, it becomes more difficult to make any design modifications because of quality, cost and schedule implications. Therefore, to facilitate to find out the best green concept alternative among a set of alternatives, in this paper, a fuzzy AHP-based FPROMETHEE II is proposed to firstly weight the evaluation criteria though F-AHP, and rank concept 
alternatives using F- PROMETHEE II. Next, this approach with three sections are explained more in detail.

\subsection{Criteria weighting through F-AHP}

The main idea of fuzzy set theory developed by Zadeh is based on an element with a degree of membership in a fuzzy set (Zadeh, 1965), which is defined by a membership function mapping elements in the universe of discourse to elements in a certain interval of $[0,1]$.

In the first section, the AHP is used for weighting a set of criteria using a nine-point scale, and based on a hierarchy considering the distribution of a goal amongst the elements being compared, and judges which element has a greater influence on that goal. It is one of the most commonly used MCDM methods, in literature and has been widely used for different kinds of MCDM problems (Ayag and Ozdemir, 2007). For weighting the evaluation criteria for green concept selection problem using F-AHP, triangular fuzzy numbers (TFNs), $\tilde{1}$ to $\tilde{9}$, are utilized to make the required pairwise comparisons of selection process to capture the vagueness of a DM.

A fuzzy number is a special fuzzy set $F=\left\{\left(x, \mu_{F}(x)\right), x \in R\right\}$, where $\mathrm{x}$ takes it values on the real line, $R:-\infty<x<+\infty$ and $\mu_{F}(x)$ is a continuous mapping from $R$ to the closed interval $[0,1]$. A TFN denoted as $\tilde{M}=(l, m, u)$, where $l \leq m \leq u$, has the following triangular type membership function. The TFNs are used to improve the traditional the nine-point scaling scheme of Saaty's to take the imprecision and vagueness of a DM judgments into consideration. In this scale; the five $\operatorname{TFNs}(\tilde{1}, \tilde{3}$, $\tilde{5}, \tilde{7}, \tilde{9})$ are defined with their membership function. All evaluation criteria and alternatives are linguistically. The shape and position of linguistically elements are chosen to illustrate the fuzzy extension of the method.

Later, the DM is asked to compare the elements at a given level on a pairwise basis to estimate their relative importance in relation to the element at the immediate proceeding level. In traditional AHP of Saaty, the required pairwise comparisons are done by using a nine-point ratio scale (Saaty, 1989). Unfortunately, although this scale has the advantages of simplicity and easiness, it is not enough to reflect the uncertainty in associated with the mapping of DM's judgment to a number. Therefore, the fuzzy logic is integrated to the conventional AHP to overcome this difficulty, called F-AHP. Next, the steps of this method is concisely given;

Step 1. Comparing the performance scores: the TFNs are used to indicate the relative strength of each pair of elements in the same hierarchy.

Step 2. Constructing the fuzzy comparison matrix: the fuzzy judgment matrix $\tilde{A}\left(a_{i j}\right)$ is constructed via pairwise comparison using TFNs as given below;

$$
\tilde{A}=\left[\begin{array}{ccccc}
1 & \tilde{a_{12}} & . . & . . & \tilde{a_{1 n}} \\
\tilde{a_{21}} & 1 & . . & . . & \tilde{a_{2 n}} \\
. . & . . & . . & . . & . . \\
\ddot{\tilde{a}} & \ddot{\tilde{a}} & . . & . . & . . \\
a_{n 1} & a_{n 2} & . . & . . & 1
\end{array}\right]
$$

where, $\tilde{a}_{i j}^{\alpha}=1$, if $i$ is equal $j$, and $\tilde{a}_{i j}^{\alpha}=\tilde{1}, \tilde{3}, \tilde{5}, \tilde{7}, \tilde{9}$ or $\tilde{1}^{-1}, \tilde{3}^{-1}, \tilde{5}^{-1}, \tilde{7}^{-1}, \tilde{9}^{-1}$, if $i$ is not equal $j$

Step 3. Solving fuzzy eigenvalue: A fuzzy eigenvalue, $\tilde{\lambda}$ is a fuzzy number solution to $\tilde{A} \tilde{x}=\tilde{\lambda} \tilde{x}$ (Eq.1), where is $n x n$ fuzzy matrix containing fuzzy numbers $\tilde{a}_{i j}$ and $\tilde{x}$ is a non-zero $n x 1$, fuzzy vector 
containing fuzzy number $x_{i}$. To perform fuzzy multiplications and additions by using the interval arithmetic and $\alpha-c u t$, the equation $\tilde{A} \tilde{x}=\tilde{\lambda} \tilde{x}$ is equivalent to

$$
\begin{aligned}
& \left\lfloor a_{i 1 l}{ }^{\alpha} x_{1 l}{ }^{\alpha}, a_{i 1 u}{ }^{\alpha} x_{1 u}{ }^{\alpha}\right\rfloor \oplus \ldots . \oplus\left\lfloor a_{i n l}{ }^{\alpha} x_{n l}{ }^{\alpha}, a_{i n u}{ }^{\alpha} x_{n u}{ }^{\alpha}\right\rfloor=\left\lfloor\lambda x_{i l}{ }^{\alpha}, \lambda x_{i u}{ }^{\alpha}\right\rfloor \quad \text { where, } \quad \tilde{A}=\left[\tilde{a_{i j}}\right], \tilde{x}^{t} \\
& =\left(\tilde{x_{1}}, \ldots ., \tilde{x}_{n}\right), \quad \tilde{a}_{i j}^{\alpha}=\left[a_{i j l}^{\alpha}, a_{i j u}^{\alpha}\right] \tilde{x}_{i}^{\alpha}=\left[x_{i l}^{\alpha}, x_{i u}^{\alpha}\right], \tilde{\lambda}^{\alpha}=\left[\lambda_{l}^{\alpha}, \lambda_{u}^{\alpha}\right] \text { for } 0<\alpha \leq 1 \text { and }
\end{aligned}
$$

all $i, j$, where $\mathrm{i}=1,2 \ldots n, j=1,2 \ldots n$ (Eq.2)

$\alpha$-cut is commonly known to incorporate a DM confidence over his/her judgments. The degree of satisfaction for a judgment matrix; $\tilde{A}$ is estimated by using the index of optimism $\mu$. The larger value of index $\mu$ indicates the higher degree of optimism. The index of optimism is a linear convex combination defined by Lee (1999) and given as the following equation: $\tilde{a_{i j}^{\alpha}}=\mu a_{i j u}^{\alpha}+(1-\mu) a_{i j l}^{\alpha}, \quad \forall \mu \in[0,1]$ (Eq.3) while $\alpha$ is fixed, the following matrix is obtained after setting the value of $\mu$, to estimate the degree of satisfaction.

$$
\tilde{A}=\left[\begin{array}{ccccc}
1 & \tilde{a_{12}} & . . & . . & a_{1 n}^{\alpha} \\
\tilde{\alpha} & & & & \tilde{\tilde{\alpha}} \\
a_{21} & 1 & . . & . . & a_{2 n}^{\alpha} \\
. . & . . & . . & . . & . . \\
. . & . . & . . & . . & . . \\
\tilde{\tilde{\alpha}} & \tilde{a_{n 1}^{\alpha}} & . . & . . & 1
\end{array}\right]
$$

The eigenvector is calculated by fixing the $\mu$ value and identifying the maximal eigenvalue. Then, the matrix is normalized and the priority weights of the concept alternatives are determined.

Step 4. Consistency analysis; To make sure that the result is based on the consistent on the judgments of the DM, first $\lambda_{\max }$ calculated by Eq.(1), then the consistency index $(\mathrm{Cl})$ is calculated for the matrix by Eq.(4). The deviations from the consistency are expressed by the $\mathrm{Cl}$; the measure of inconsistency; $C I=\frac{\lambda_{\max }-n}{n-1}$ (Eq.4); Later, the consistency ratio (CR) is calculated by Eq. (5) by dividing the value of $\mathrm{Cl}$ by the value from the table of Random Consistency Index (RI), the average index for randomly generated weights based on the matrix size (Saaty, 1981); $C R=\frac{C I}{R I}$ (Eq.5). For consistency for a matrix, the value of CR should be less than 0.10 , and it means that the pairwise comparisons of the DM are consistent and acceptable, otherwise not.

\subsection{Ranking alternatives through the three F-PROMETHEE II}

In literature; it is reported that the PROMETHEE II has been used with success to solve various MCDM problems (Samanlioglu and Ayag, 2016). It is based on a comparison pair per pair of possible decisions along each criterion. Possible decisions are evaluated according to different criteria, which have to be maximized or minimized. It also requires two additional types of information for each criterion; a weight and a preference function. The preference function characterizes the difference for a criterion between the evaluations obtained by two possible decisions into a preference degree in the interval of $[0,1]$. To facilitate the definition of these functions, six basic preference functions were proposed by Figueira et. al. (2004). Next, the four steps of F-PROMETHEE II are presented (Samanlioglu and Ayağ, 2016); 
Step 1. Construct a fuzzy decision making matrix together with the results of the F-AHP method; $W=\left(w_{1}, w_{2}, w_{3}, \ldots, w_{n}\right)$, where $\left.\sum w_{i}=1(i=1,2, \ldots, n)\right)$, and a typical $m$ by $n$ fuzzy decision matrix is shown as below;

$$
\begin{aligned}
& \left(w_{1} \ldots w_{j} \ldots w_{n}\right) \\
& \left(\hat{c}_{1} \ldots \hat{c}_{j} \ldots \hat{c}_{n}\right)
\end{aligned}
$$

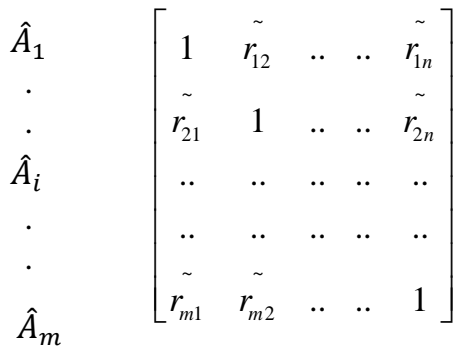

Here, $\widehat{c_{j}} \in \widehat{C}$ is a fuzzy positive criterion. The criterion is a maximum criterion, if the DM prefers more value for it. Otherwise, it is a minimum. $\widehat{A_{l}} \in \widehat{A}$ is fuzzy alternative. $\widehat{A^{*}}$ is the fuzzy alternative from $\hat{A}$. $\widehat{r_{l j}} \in \hat{r}$ is the utility value. $w_{j} \in W$ is the weight of $\widehat{c_{j}}$.

Step 2. Index fuzzy numbers in the fuzzy decision matrix: the fuzzy number in the fuzzy matrix is defuzzified with centroid defuzzification approach (Wang, 2009) to the crisp number by Eq. (6);

$$
(l, m, u)=(l+m+u) / 3
$$

In other words, the above process converts a fuzzy decision matrix into a crisp decision matrix as follows:

$$
\begin{aligned}
& \left(w_{1} \ldots w_{j} \ldots w_{n}\right) \\
& \left(c_{1} \ldots c_{j} \ldots c_{n}\right) \\
& \begin{array}{l}
A_{1} \\
\cdot \\
\cdot \\
A_{i} \\
\cdot \\
\cdot \\
A_{m}
\end{array}\left[\begin{array}{ccccc}
1 & r_{12} & . . & . . & r_{1 n} \\
r_{21} & 1 & . . & . . & r_{2 n} \\
. . & . . & . . & . . & . . \\
. . & . . & . . & . . & . . \\
r_{m 1} & r_{m 2} & . . & . . & 1
\end{array}\right]
\end{aligned}
$$

where, $c_{j} \in C$ is the positive criterion, $A_{i} \in A$ is the alternative, $A^{*}$ is the ideal alternative from $A, r_{i j} \in r$ is the utility value, $w_{j} \in W$ is the weight of $c_{j}$. The cap removal from the notations is crisp value.

Step 3. Calculate aggregated preference indices: $P_{j}\left(A_{i}, A_{k}\right)=P_{j}\left(d\left(A_{i}, A_{k}\right)\right)=P_{j}\left(r_{i j}-r_{k j}\right)$ is a preference function showing how much $A_{i}$ prefers to $A_{k}$ with respect to $c_{j}$. Brans et al. (1984) defined the six types of generalized functions, and also pointed out that the Gaussian criterion rather than the others was mostly prefered by users for practical applications especially in the case of continuing data. Due to the fact that the evaluation criteria contain continuing data, the Gaussian criterion preference function was chosed here for the evaluation process as given as follows: 


$$
P(d)=\left\{\begin{array}{cc}
0 & d \leq 0 \\
1-e^{-\frac{d^{2}}{2 s^{2}}} & d>0
\end{array}\right\}
$$

if the criterion is a maximum and

$$
P(d)=\left\{\begin{array}{cc}
0 & d \geq 0 \\
1-e^{-\frac{d^{2}}{2 s^{2}}} & d<0
\end{array}\right\}
$$

if the criterion is a minimum.

Aggregated preference index $\pi\left(A_{i}, A_{k}\right)$ expresses the degree of how much $A_{i}$ is preferred to $A_{k}$ over all the criteria. The aggregated preference indices are of the form:

$$
\pi\left(A_{i}, A_{k}\right)=\frac{\sum_{j=1}^{n} P_{j}\left(A_{i}, A_{k}\right) \cdot w_{j}}{\sum_{j=1}^{n} w_{j}}, \forall A_{i}, A_{k} \in A \text { and } i \neq k
$$

Step 4. Calculate outranking flow. Each alternative $A_{i}$ is facing $(m-1)$ other alternatives in $A$. In order to rank the alternatives, the outranking flows are defined as follows:

The positive outranking flow is of the form:

$$
\emptyset^{+}\left(A_{i}\right)=\sum_{k=1}^{m} \pi\left(A_{i}, A_{k}\right)
$$

The negative outranking flow is of the form:

$$
\emptyset^{-}\left(A_{i}\right)=\sum_{k=1}^{m} \pi\left(A_{k}, A_{i}\right)
$$

The net outranking flow is applied and is in the form of:

$$
\emptyset\left(A_{i}\right)=\emptyset^{+}\left(A_{i}\right)-\emptyset^{-}\left(A_{i}\right), \forall i \in\{1, \ldots, m\}
$$

The positive outranking flow expresses how an alternative $\boldsymbol{A}_{\boldsymbol{i}}$ is outranking all the others. Higher $\emptyset^{+}\left(\boldsymbol{A}_{\boldsymbol{i}}\right)$ gives a better alternative. On the other hand, the negative outranking flow expresses how an alternative $\boldsymbol{A}_{\boldsymbol{i}}$ is outranked by all the others. The lower $\emptyset^{-}\left(\boldsymbol{A}_{\boldsymbol{i}}\right)$ gives a better alternative. The higher $\emptyset\left(\boldsymbol{A}_{i}\right)$ specifies the final better alternative.

\section{Case study}

In the previous section, a F-AHP-based F-PROMETHEE II has been presented to evaluate a set of green conceptual design alternatives in terms of the evaluation criteria in a NPD environment. In this section, a case study is presented for potential readers or practitioners to clearly explain of how the comparative approach works on a real-life case. For this purpose; the case study is constructed by inspiring from a study previously done in a hot runner system manufacturer in Canada (Ayag, 2014). This case study has the four different concepts, named; Concept $A, B, C$ and $D$ respectively, together with the four-evaluation criteria as given in table 1, three of which were determined by utilizing the previous study, last one; green criterion was newly-added by taking the principles of Design for Environment (DfE) into consideration in order to obtain environmental-friendly products, which are so vital and expected by most of mold-manufacturers in today's business world.

Table 1. List of criteria for the green concept selection problem 


\begin{tabular}{cll}
\hline Code & Criteria & Definition \\
\hline C & Cost & Development cost, unit manufacturing cost \\
R & Risk & Envisioning risk, design risk, execution risk, on-time delivery \\
Q & Quality & $\begin{array}{l}\text { Product quality, cycle time, quick color change, precision, flexibility, } \\
\text { conductivity, strength, resistance, repeatability and reproducibility }\end{array}$ \\
G & Green & $\begin{array}{l}\text { Environmental friendly materials and production, amount of recycling } \\
\text { content, environmental friendly use of product and sustainable } \\
\end{array}$ \\
& & packaging, disposability at the end of the product life \\
\hline
\end{tabular}

\section{Determining weights of the criteria through F-AHP}

Firstly, after weighting the four-evaluation criteria; cost, risk, quality, and green, the details of which are given in table 3 , the TFNs $(\tilde{1}, \tilde{3}, \tilde{5}, \tilde{7}, \tilde{9})$ are used to express the preference in the pairwise comparisons, and the fuzzy pair-wise comparison matrix $(\tilde{A})$ for the relative importance of the criteria is constructed as given in table 2 .

Table 2. Fuzzy comparison matrix of the criteria using TFNs

\begin{tabular}{lcccc}
\hline Criteria & Cost & Risk & Quality & Green \\
\hline Cost & 1 & $\tilde{3}$ & $\tilde{9}$ & $\tilde{9}$ \\
Risk & $\tilde{3}$ & 1 & $\tilde{3}$ & $\tilde{7}$ \\
Quality & $\tilde{3^{-1}}$ & $\tilde{3^{-1}}$ & $\tilde{1}$ & $\tilde{1}$ \\
Green & $\tilde{9^{-1}}$ & $\overline{7}^{-1}$ & $1^{-1}$ & 1 \\
\hline
\end{tabular}

Secondly, the lower and upper limits of the fuzzy numbers in the fuzzy matrix $(A)$, with respect to $\alpha$, the confidence level are defined by applying Eq. (2) as follows:

$\tilde{1}_{\alpha}=[1,3-2 \alpha], \tilde{3}_{\alpha}=[1+2 \alpha, 5-2 \alpha], \tilde{3}_{\alpha}{ }^{-1}=\left[\frac{1}{5-2 \alpha}, \frac{1}{1+2 \alpha}\right], \tilde{5}_{\alpha}=[3+2 \alpha, 7-2 \alpha]$,

$\tilde{5}_{\alpha}{ }^{-1}=\left[\frac{1}{7-2 \alpha}, \frac{1}{3+2 \alpha}\right], \tilde{7}_{\alpha}=[5+2 \alpha, 9-2 \alpha], \tilde{7}_{\alpha}{ }^{-1}=\left[\frac{1}{9-2 \alpha}, \frac{1}{5+2 \alpha}\right], \tilde{9}_{\alpha}=[7+2 \alpha, 11-2 \alpha]$,

$\tilde{9}_{\alpha}^{-1}=\left[\frac{1}{11-2 \alpha}, \frac{1}{7+2 \alpha}\right]$

Then, the values of $\alpha=0.5$ and $\mu=0.5$ were determined using the interval of [0-1] by the DM, who works as a design engineer at the company, are substituted, where $\mu$ indicates the coefficient of optimism, above expression into the fuzzy comparison matrix, and the $\alpha$-cuts fuzzy comparison matrix is obtained by Eq. (3) as presented in table 3.

Table 3. $\alpha$-cuts fuzzy comparison matrix for the criteria $(\alpha=0.5, \mu=0.5)$

\begin{tabular}{lcccc}
\hline Criteria & Cost & Risk & Quality & Green \\
\hline Cost & 1 & {$[2,4]$} & {$[8,10]$} & {$[8,10]$} \\
Risk & {$[1 / 4,1 / 2]$} & 1 & {$[2,4]$} & {$[6,8]$} \\
Quality & {$[1 / 10$,} & {$[1 / 4,1 / 2]$} & 1 & {$[1,2]$} \\
Green & {$[1 / 10$,} & {$[1 / 8,1 / 6]$} & {$[1 / 2,1]$} & 1 \\
\hline
\end{tabular}

Later, the eigenvalue of the matrix $A$ is calculated by solving the characteristic equation of $A$, $\operatorname{det}(A-\lambda I)=0$, and found out all $\lambda$ values for $A\left(\lambda_{1}, \lambda_{2}, \lambda_{3}\right)$. Next, the largest eigenvalue of pairwise matrix, $\lambda_{\max }$, is calculated by using Eq. (1), where the matrix size, $n$ is 4 , and the $R I(4)$ is 1.12 . Finally, 
the $C l$ and the $C R$ of the matrix $A$ are calculated by Eq. (4) and Eq. (5) and given in table 4. As seen in table, the $C R$ value, 0.052 is less than to 0.10 , and it means that all the pairwise comparisons of the DM are consistent. As also seen in the far right column of the table, the e-Vector of the criteria weights as crisp values are respectively as follows; $\mathrm{W}=(0.607,0.263,0.077,0.053)$.

Table 4. Eigenvector for comparison matrix of the criteria $(C R=0.052)$

\begin{tabular}{lccccc}
\hline Criteria & Cost & Risk & Quality & Green & \\
\hline Cost & 1.000 & 3.000 & 9.000 & 9.000 & 0.607 \\
Risk & 0.375 & 1.000 & 3.000 & 7.000 & 0.263 \\
Quality & 0.113 & 0.375 & 1.000 & 1.500 & 0.077 \\
Green & 0.113 & 0.146 & 0.750 & 1.000 & 0.053 \\
\hline & & & & $\lambda_{\text {Cqx }}$ & 4.174 \\
& & & & $R I$ & 0.058 \\
& & & & $C R$ & 1.12 \\
& & & & & \\
& & &
\end{tabular}

\section{Ranking green concept alternatives using F-PROMETHEE II}

In the previous section, the relative weights of the evaluation criteria are determined, and next, the fuzzy PROMETHEE II for ranking green concept alternatives are implement;

First, the vector of criteria weights $(W)$ and fuzzy decision matrix $(\tilde{X})$ are given to the alternatives with respect to all the criteria; cost, risk, quality, and green as shown in table 6. Moreover, the values of $s$ in the table indicate maximum due to the fact that each criterion is maximum with the value of $s$; being equals to 5. For example: If the alternatives; Concept $A$, Concept $B$, Concept $C$, and Concept $D$ are evaluated in terms of the criterion; Cost, using the TFNs, the fuzzy values; $\{(7.0,8.0,9.0),(4.0,5.0$, $6.0),(2.0,3.5,5.0),(1.0,2.0,3.0)\}$ are obtained respectively.

Later, the fuzzy decision matrix is converted into crisp decision matrix by Eq. (6) as shown in table 7 . With respect to the crisp decision matrix in table 6, the aggregated preference matrix for $P 1$ (Concept $A$, Concept $B$ ) is shown in table 7.

\begin{tabular}{lcccc}
\multicolumn{6}{l}{ Table 6. Fuzzy decision matrix } & \multicolumn{4}{c}{ for } & \multicolumn{3}{c}{ green concept selection } & for & F-PROMETHEE II \\
\hline Criteria & Cost & Risk & Quality & Green \\
Value & Max. & Max. & Max. & Max. \\
s & 5 & 5 & 5 & 5 \\
Weight & 0.607 & 0.263 & 0.077 & 0.053 \\
\hline Concept A & $(7.0,8.0$, & $(2.0,3.5,5.0)$ & $(2.0,3.5$, & $(5.0,6.5,8.0)$ \\
Concept B & $(4.0,5.0$, & $(4.0,5.0,6.0)$ & $(1.0,2.0$, & $(8.0,9.0$ \\
Concept & $(2.0,3.5$, & $(8.0,9.0$, & $(5.0,6.5$, & $(7.0,8.0,9.0)$ \\
Concept & $(1.0,2.0$, & $(2.0,3.5,5.0)$ & $(4.0,5.0$, & $(2.0,3.5,5.0)$ \\
\hline
\end{tabular}

\begin{tabular}{lcccc}
\multicolumn{5}{c}{ Table 7. Decision making matrix after indexing } \\
\hline Criteria & Cost & Risk & Quality & Green \\
Value & Max. & Max. & Max. & Max. \\
S & 5 & 5 & 5 & 5 \\
\hline Weight & 0.607 & 0.263 & 0.077 & 0.053 \\
Concept A & 8.000 & 3.500 & 3.500 & 6.500 \\
Concept B & 5.000 & 5.000 & 2.000 & 9.000 \\
Concept C & 3.500 & 9.000 & 6.500 & 8.000 \\
Concept D & 2.000 & 3.500 & 5.000 & 3.500 \\
\hline
\end{tabular}

The Gaussian criterion function is chosen for all the criteria where the parameter $s$ for each criterion is the value of 5 . To show the calculation steps of how the values in table 8 are obtained, the following example can be given as follows: If the alternative Concept $A$ is compared with the alternative Concept $B$, the related the values $x_{1}, y_{1}$ for $P 1$ (Concept $A$, Concept $B$ ) are calculated using the data in table 8 by Eq. (7) and Eq.(8) as given below;

$x_{1}=8.00-5.00=3.00, y_{1}=1-e^{\left(-\left(x_{1}^{2}\right) /\left(2 * s^{2}\right)\right.}=1-e^{\left(-\frac{3.000^{2}}{2 * 5^{2}}\right)}=0.1647, \mathrm{z}=\sum_{i=1}^{4} w_{i} * y_{i}=0.10338$ 
In addition; the $z$ value is found after determining all the values of $\boldsymbol{x}_{\boldsymbol{i}}, \boldsymbol{y}_{i}$ for $P_{i}(i=1,2,3,4)$ as the number of the concept alternatives. The results of the all the elements are given in table 9.

Table 8. Calculation steps of each element of aggregated preference index matrix for P1 (Concept $A$, Concept $B$ )

\begin{tabular}{lcccc}
\hline Pairwise Comparison & $w_{i}$ & $x_{i}$ & $y_{i}$ & $\mathrm{z}$ \\
\hline P1 (Concept A, Concept B) & 0.607 & 3.000 & 0.1647 & \\
P2 (Concept A, Concept B) & 0.263 & -1.500 & 0.0000 & 0 \\
P3 (Concept A, Concept B) & 0.077 & 1.500 & 0.0440 & 0.10338 \\
P4 (Concept A, Concept B) & 0.053 & -2.500 & 0.0000 & \\
\hline
\end{tabular}

Table 9. Aggregated preference index matrix

\begin{tabular}{lcccc}
\hline Alternatives & Concept A & Concept B & Concept C & Concept D \\
\hline Concept A & 0 & 0.10338 & 0.20215 & 0.31154 \\
Concept B & 0.01780 & 0 & 0.02776 & 0.13562 \\
Concept C & 0.13440 & 0.09767 & 0 & 0.16713 \\
Concept D & 0.00339 & 0.01268 & 0.00000 & 0 \\
\hline
\end{tabular}

Later, by using the aggregated preference index matrix, the positive, negative and net outranking flows for each alternative are calculated by Eq. (9-10-11-12) and presented in table 10. As seen in table, the best alternative is Concept $A$ and the ranking is found as; Concept $A$-Concept $C$-Concept $B$-Concept $D$.

Table 10. Outranking flow indices and rank through F-PROMETHEE II

\begin{tabular}{ccccc}
\hline Alternatives & Concept A & Concept B & Concept C & Concept D \\
\hline$\emptyset^{+}$ & 0.61707 & 0.18118 & 0.39919 & 0.01607 \\
$\emptyset^{-}$ & 0.15559 & 0.21373 & 0.22990 & 0.61429 \\
$\varnothing$ & 0.46148 & -0.03255 & 0.16929 & -0.59822 \\
Ranking & 1 & 3 & 2 & 4 \\
\hline
\end{tabular}

\section{Conclusions}

The objective of the research was, to propose a F-AHP based F-PROMETHEE II approach to green concept selection problem. As the F-AHP is used to weight evaluation criteria, the F-PROMETHEE II are respectively used for ranking the concept alternatives, and determine the best concept alternative. Furthermore, the case study was inspired from the previous work of the author, which was realized in a hot runner systems manufacturer, used in injection molding systems in a Canada. In a new product development process, the back- and front- ends of development efforts mainly affects to determining the following criteria; cost, risk, quality, and green used in this paper. On the other hand, the F-MCDM methods have the following limitations; for instance; because the result (or ranking) of any method depends on the judgments of a DM. The possibility of bias of the DM to any particular alternative cannot be easily managed as especially in the F-AHP, because inconsistency value might lead to wrong results. Inclusion of environmental-related criteria into concept selection problem has been gaining increasing importance last decade.

\section{References}

Ayag, Z. (2005a). An integrated approach to evaluating conceptual design alternatives in a new product development environment. International Journal of Production Research, 43(4), 687-713. https://doi.org/10.1080/00207540512331311831

Ayag, Z. (2005b). A fuzzy AHP-based simulation approach to concept evaluation in a NPD environment. IIE Transactions, 37, 827-842. https://doi.org/10.1080/07408170590969852

Ayag, Z., Ozdemir, R.G. (2007). An analytic hierarchy process-based approach to concept evaluation in a new product development environment", Journal of Engineering Design, 18, 209-226. https://doi.org/10.1080/09544820600752740

Ayag, Z. (2014). An integrated approach to concept evaluation in a new product development, Journal of Intelligent Manufacturing. DOI: 10.1007/s10845-014-0930-7 (article-in-press). 
Brans, J.P., Vincke, Ph. and Marechal, B. (1986). How to select and how to rank projects: The PROMETHEE method, European Journal of Operational Research, 24, 228- 238. https://doi.org/10.1016/0377-2217(86)90044-5

Duffy, A.H.B., Andreasen, M. M., Maccallum, K. J. and Reijers, L.N. (1993). Design co-ordination for concurrent engineering. Journal of Engineering Design, 4, 251-261. https://doi.org/10.1080/09544829308914785

Figueira, J., Greco, S. and Ehrgott, M. (2004). Multiple Criteria Decision Analysis: State of the Art Surveys, Springer, New York. https://doi.org/10.1007/b100605

Lee, A.R. (1999) Application of modified fuzzy AHP method to analyze bolting sequence of structural joints. UMI Dissertation Services, A Bell \& Howell Company.

Saaty, T. L. (1981). The Analytical Hierarchy Process. McGraw Hill: New York

Saaty, T. L. (1989). Decision making, scaling, and number crunching. Decision Science, 20, 404-409. https://doi.org/10.1111/j.1540-5915.1989.tb01887.x

Samanlioglu, F., Ayağ, Z. (2016). A fuzzy ANP-based PROMETHEE II approach for evaluation of machine tool alternatives, Journal of Intelligent and Fuzzy Systems, 30, 2223-2235. https://doi.org/10.3233/IFS-151991

Ulrich, K.T. and Eppinger, S.D. (2000). Product Design and Development, Second Edition. McGraw-Hill, New York.

Wang, Y. (2009). Centroid defuzzification and the maximizing set and minimizing set ranking based on alpha level sets, Computers and Industrial Engineering, 57, 228-236. https://doi.org/10.1016/j.cie.2008.11.014

Zadeh, L. A. (1965). Fuzzy sets. Information and Control, 8, 338-353. https://doi.org/10.1016/S00199958(65)90241-X

Zhang, H.C., Kuo, T.C., and Lu, H. (1997). Environmentally conscious design and manufacturing: a state-of-the-art survey, Journal of Manufacturing Systems, 16, 352-371. https://doi.org/10.1016/S0278-6125(97)88465-8 